\title{
PERFIL DEL COMMUNITY MANAGER EN LAS AGENCIAS DE PUBLICIDAD Y RELACIONES PÚBLICAS DE ESPAÑA
}

\author{
Proffle of the community manager in advertising and \\ public relations agencies in Spain
}

\section{Carmen Silva-Robles}

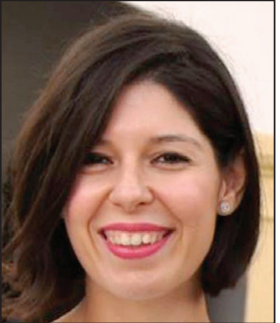

Carmen Silva-Robles es doctora en publicidad y relaciones públicas y licenciada en periodismo por la Universidad de Sevilla. Pertenece a SEJ536. Laurea, equipo de investigación en relaciones públicas, ceremonial y protocolo. Sus principales líneas de investigación son los perfiles de comunicación corporativa y la comunicación de marcas premium. Es docente del Departamento de Marketing y Comunicación de la Universidad de Cádiz y consultora en los estudios de comunicación de la Universitat Oberta de Catalunya. http://orcid.org/0000-0001-7993-8760

Universidad de Cádiz, Facultad de Ciencia Sociales y de la Comunicación Av. de la Universidad, s/n. 11405 Jerez de la Frontera (Cádiz), España. carmensilva.robles@uca.es csilvaro@uoc.edu

\section{Resumen}

El community manager ha sido una de las nuevas profesiones más destacadas durante los primeros años de vida de la web 2.0. Con el tiempo tanto el puesto profesional como las funciones que ejerce se han consolidado y normalizado en el panorama de la comunicación corporativa. Por ello no pocas organizaciones contratan este servicio a agencias. Se analiza cómo son los profesionales que ejercen de community manager en las agencias de publicidad y relaciones públicas españolas. A través de una encuesta se ha descrito el perfil de las personas que realizan esta función. Los resultados arrojados nos muestran una mujer de entre 25 y 35 años, que ha estudiado periodismo y con una experiencia previa de 2 ó 3 años en medios digitales. Estamos por tanto ante una profesional joven y con un CV ligado al mundo de la comunicación.

\section{Palabras clave}

Community management; Agencias publicidad y relaciones públicas; Comunicación corporativa; Comunicación digital; Web 2.0; Relaciones públicas; Profesionales de la comunicación; España.

\section{Abstract}

Community management has emerged as a new profession since the advent of web 2.0. Professional positions and functions have been consolidated and standardized within corporate communication; consequently, many organizations hire community management services or agencies. This paper discusses the roles of the professionals who are responsible for community management in Spanish advertising and public relations agencies. A survey identified the typical profile of a professional in this field: a woman, aged 25-35 years, journalism degree, with two to three years previous digital media experience. In other words, a young professional with a CV linked to the world of communication.

\section{Keywords}

Community management; Advertising agencies; Public relations agencies; Corporate communication; Digital communication; Web 2.0; Public relations; Communication professionals; Spain.

Silva-Robles, Carmen (2016). "Perfil del community manager en las agencias de publicidad y relaciones públicas de España". El profesional de la información, v. 25, n. 2, pp. 237-245.

http://dx.doi.org/10.3145/epi.2016.mar.10

\section{Estado de la cuestión}

El sector TIC -tecnologías de la información y de la comunicación- es en la actualidad una de las áreas con mejores opciones de trabajo. La llegada de las redes sociales ha propi- ciado la creación de nuevos puestos que amplía la demanda de trabajadores especializados por parte de las empresas. Contar con profesionales formados en competencias digitales es una necesidad para ellas. 
Así se pone de manifiesto en el estudio Perfiles profesionales más demandados en el ámbito de los contenidos digitales en España 2012-2017 (FTI, 2011), que realiza una estimación de los puestos de trabajo en ese ámbito que están siendo requeridos y que tendrán mayor demanda en los próximos años. Para cada perfil se identifican formación, competencias y habilidades, experiencia, funciones y tareas, relaciones con otros puestos o perfiles, y empleabilidad. El estudio revela que el sector de la comunicación es uno de los principales motores de estos nuevos puestos profesionales.

El informe Observatorio del mercado laboral de los profesionales del marketing, la comunicación y la publicidad digital (2- oleada, junio 2011) elaborado por IAB Spain (Interactive Advertising Bureau) describe cómo son las personas que están desempeñando las funciones de comunicación digital y cuál será el futuro de estos puestos de trabajo. Destaca la figura del gestor de comunidades online o community manager (en adelante, CM), con una demanda del $43 \%$ del total de puestos.

\section{El sector TIC mantiene una alta tasa de empleabilidad, las empresas necesitan profesionales capaces de gestionar nue- vas funciones desde una perspectiva di- gital}

A pesar de la importancia que ha adquirido el $C M$, son pocas las publicaciones científicas que lo han tratado. Uno de los principales referentes es el análisis anual Les community managers en France, que lleva a cabo desde 2010 la empresa gala RegionJob. En la última edición (RegionJob, 2014), elaboró una encuesta de 70 preguntas distribuida entre 760 CM mediante la cual se obtuvieron datos sobre la vida profesional, salarios, herramientas de trabajo y perfiles de los CM que trabajan por cuenta propia y ajena.

En esta misma línea se encuentran los Community managers reports de SocialFresh en Estados Unidos, realizados en 2011, 2012 y 2013 a partir de una encuesta a 1.000 profesionales (Keath, 2013).

En España hay varias aproximaciones interesantes, como las de Castelló-Martínez (2010a; 2010b; 2012), que ahonda en esta figura desde el prisma de la publicidad. Por su parte, Cobos (2011) define la profesión y revisa las funciones y herramientas que emplean. Una línea que continúa GodoyMartín (2012), que analiza funciones y herramientas, ciñéndose a los CM que trabajan en la administración pública andaluza. Almansa-Martínez y Godoy-Martín (2012) repiten este análisis entre los $C M$ de las principales empresas españolas que cotizan en el Ibex 35.

Sanz-Martos (2012), desde el punto de vista de la biblioteconomía y documentación, identifica los perfiles profesionales nacidos con la web social, describiendo sus principales funciones, competencias y su peso en las organizaciones. Silva-Robles (2012), reflexiona sobre la función directiva de este puesto, desde la perspectiva de las relaciones públicas. Elorriaga-Illera (2013) analiza los CM del País Vasco y Navarra, destacando los datos sobre las herramientas que emplean, el tipo de relación que mantienen con las empresas y los sectores empresariales que cuentan con esta figura.

Frente a estas publicaciones, la presente investigación parte de la idea de que community management es una labor de comunicación corporativa, y centra su interés en la figura y función de $C M$ en el ámbito concreto de las agencias de publicidad y relaciones públicas.

Noguero-Grau (1995, pp. 45-62) identifica tres tipos de sujetos participantes en los procesos de relaciones públicas:

- promotores: organizaciones que deciden llevar a cabo acciones de relaciones públicas para conseguir o facilitar la consecución de determinados objetivos;

- ejecutores: profesionales que se ocupan de planificar y ejecutar las acciones de relaciones públicas para los sujetos promotores;

- públicos: actores sociales con los que los sujetos promotores desean armonizar sus intereses y puntos de vista.

Los sujetos promotores disponen de dos grandes vías para el desarrollo de las actividades de relaciones públicas: la interna y la externa. Es decir, mediante un departamento interno de comunicación o mediante la contratación de una agencia especializada (Castellblanque, 2001). Los profesionales de una agencia ofrecen una visión externa que suele ser muy útil para las empresas, puesto que será más independiente y fresca que la que aportan sus trabajadores (Aced, 2013, p. 51). Por ello, entendiendo community management como una labor de comunicación que se externaliza como otras funciones, analizamos los sujetos ejecutores que la realizan; en este caso los $C M$.

\section{El CM de agencia es una mujer de entre} 25 y 35 años, que ha estudiado periodismo y con una experiencia previa de 2 ó 3 años en medios digitales

Determinar la relación de community management con un área de estudio o de trabajo es un punto conflictivo sobre el que los investigadores tienen puntos de vista diferentes. Las opiniones más enfrentadas son las que contraponen marketing y comunicación. En el trabajo realizado por Elorriaga-Illera (2013) entre los gestores de comunidades de empresas del País Vasco se contabiliza un $48 \%$ de casos procedentes de comunicación -sin precisar el área de especialización-. Sin embargo la autora considera al CM como un perfil de marketing por las funciones que lleva a cabo puesto que las contempla desde la perspectiva del fin último de la venta. Una idea en la que coincide con Perlado:

"La vinculación de la figura del community manager al marketing empresarial es palmaria y aleja esta figura de las exigencias informativas de las audiencias. El community manager no es un periodista sino un profesional del marketing" (Perlado en Diezhandino, 2012, p. 103).

En contraposición a estas opiniones, Cobos (2011, p. 6) señala: 
“Actualmente, el perfil profesional que suele ser más solicitado para los cargos de community manager es el de periodista/comunicador social. La razón es que en su formación universitaria, estos profesionales adquieren conocimientos y desarrollan las habilidades que se requieren: redacción y ortografía, relaciones públicas, periodismo digital, medios de comunicación, entre otros".

De este modo acerca el $C M$ a la comunicación y especialmente a los periodistas y añade que "para los egresados de estos programas se convierte en una nueva opción laboral por explorar" (Cobos, 2011, p. 6).

Las opiniones más enfrentadas sobre el $C M$ son aquellas que contraponen el perfil de marketing al de comunicación

Este conflicto no deja de ser el mismo que históricamente ha existido con la comunicación corporativa y el marketing. En el caso de los directores de comunicación, El estado de la comunicación España (Dircom, 2010, p. 4) revela que el $35,3 \%$ de los dircoms -directores de comunicación- españoles es licenciado en periodismo, el 32,6\% ha cursado estudios de tercer ciclo -el 35,94\% de los profesionales encuestados para este proyecto también tenían estudios de tercer ciclo- el 5,3\% es licenciado en publicidad y relaciones públicas, y el $1,7 \%$ ha estudiado marketing.

Centrándonos en los $C M$, tenemos datos sobre los que trabajan en las instituciones públicas andaluzas. El 41,9\% tenía titulación de periodismo, el 4,7\% publicidad y relaciones públicas y un $30 \%$ otros estudios, entre ellos informática, economía, sociología, información y documentación, estadística o ciencias políticas (Godoy-Martín, 2012, p. 63). La investigación realizada entre los CM de las empresas del Ibex 35 también coincide: el $60 \%$ de ellos es periodista, frente a un $5 \%$ de publicistas y un $10 \%$ procedente de comunicación audiovisual (Almansa-Martínez; GodoyMartín, 2012, p. 61).

Estos datos se contradicen con los publicados por la IAB (2011) que afirma que la carrera cursada en mayor proporción por los profesionales de la comunicación y el marketing es publicidad y relaciones públicas (23\%).

Respecto a la experiencia profesional previa, en los casos estudiados por Castelló-Martínez (2010b, p. 91) se habla de al menos tres años de experiencia $(38,4 \%)$. El análisis realizado por Elorriaga-Illera también rebaja la experiencia de los CM; en un $84 \%$ de sus casos es la primera vez que trabajan como tal.

En cuanto al género de los profesionales de la comunicación corporativa, el estudio de Adecec (2008) confirmaba la existencia de un mayor número de mujeres trabajando en agencias y departamentos de comunicación. Entendemos que esta mayoría se repite entre los $C M$ españoles. Así ocurre en Francia según los datos ofrecidos por Les community managers en France 2014, donde el $55 \%$ son mujeres.

\section{Objetivos e hipótesis de investigación}

Esta investigación se centra en hacer una radiografía de los CM de las agencias de comunicación españolas y sus características principales. Por ello nos planteamos como objetivos:

01. Determinar sexo, edad, formación, y currículum vitae de los CM.

O2. Describir su trayectoria profesional tipo.

Partimos de las siguientes hipótesis:

HP: Los CM de agencias tienen un perfil similar al de los ejecutivos de cuentas.

HS: Los CM de agencias son mayoritariamente mujeres jóvenes (menores de 35 años) que han estudiado periodismo y tienen una experiencia profesional previa en el mundo de la comunicación digital.

\section{Metodología}

Se hizo una encuesta entre los profesionales que gestionan comunidades de organizaciones en agencias de comunicación.

Este método permite obtener información de manera clara y precisa, existe un formato estandarizado de preguntas y el informante reporta sus respuestas. Los cuestionarios sirven para entregar descripciones de los objetos de estudio, detectar patrones y relaciones entre las características descritas. Las encuestas resultan especialmente efectivas en investigaciones donde, como es el caso que nos ocupa, se busca generalizar el resultado a una población definida (Buendía-Eisman; Colás-Bravo; Hernández-Pino, 1998).

Nos centramos en dos tipos de agencias:

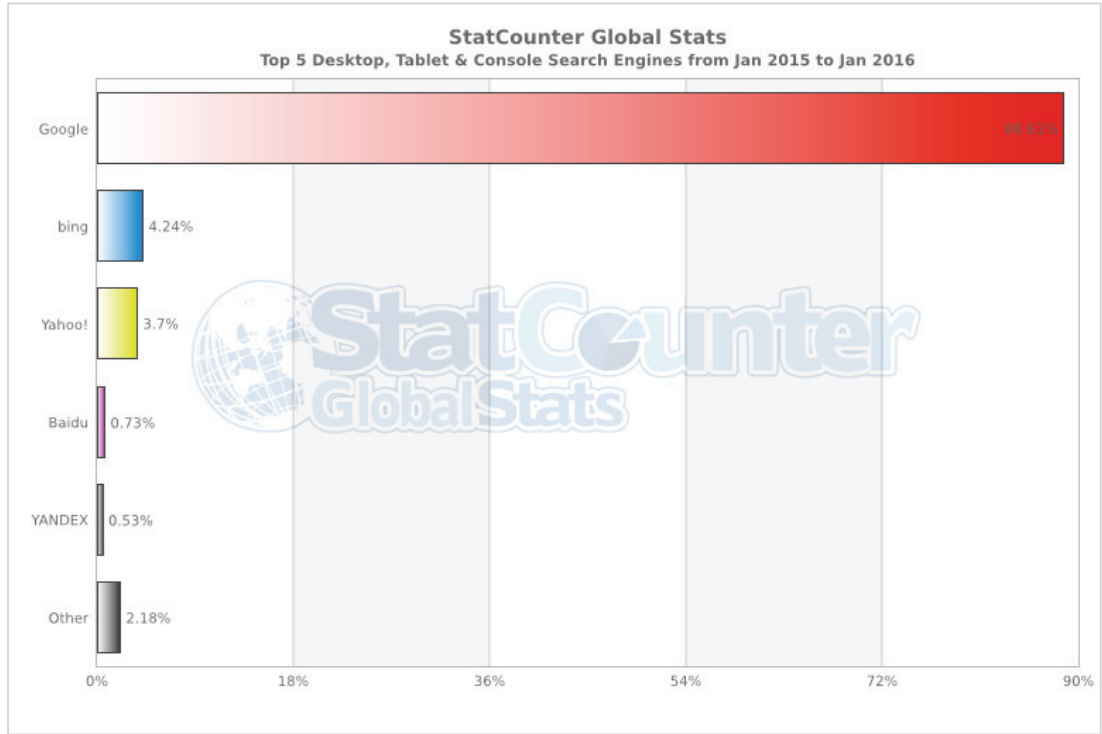

Figura 1. Top 5 de buscadores utilizados en España en 2014. Búsquedas realizadas desde ordenador de mesa, tabletas y consolas.

Fuente: StatCounter Global Stats

http://gs.statcounter.com/\#search_engine-ww-monthly-201501-201601-bar 


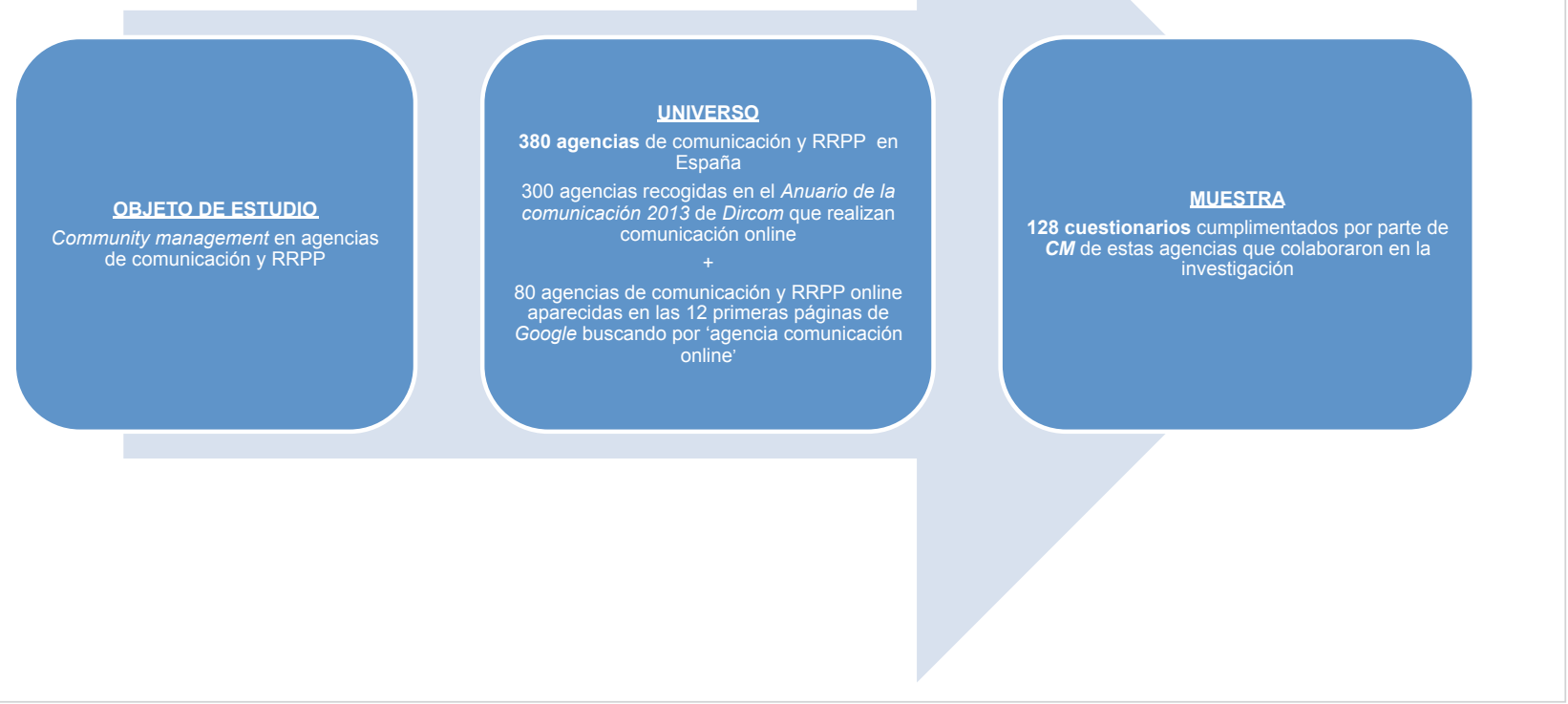

Figura 2. Muestra para la encuesta

- las que aparecen en el Anuario de la comunicación 2013, bajo el epígrafe "Empresas de comunicación y RRPP". Esta publicación, emitida anualmente por la Asociación de Directores de Comunicación de España (Dircom), recoge los datos de las agencias asociadas;

- agencias de comunicación online españolas: no se localizó ningún directorio que compile este tipo de empresas, por lo que se seleccionaron las que aparecían en las 12 primeras páginas del buscador Google al realizar la búsqueda "agencia comunicación online". Se usó comunicación en lugar de relaciones públicas por entenderse como una etiqueta más amplia y utilizada. Se empleó Google por ser el buscador más utilizado por los españoles como demuestra el ranking de Statcounter. La recolección de las agencias que aparecían a través del buscador se paró en la página 12 puesto que a partir de la 13 dejaron de mostrarse empresas con sede en España.

La población de estudio contó un total de 380 agencias, 300 pertenecientes a la asociación Dircom y 80 online, de las que se obtuvieron 128 encuestas respondidas. Dentro de las agencias se localizó a las personas que trabajan como CM o hacen las labores de community management y a ellas se les envió la encuesta.

\subsection{Diseño de la herramienta de investigación}

Primero se realizó una prueba piloto enviando cuatro cuestionarios durante el mes de febrero de 2014. Con las respuestas y comentarios recibidos se elaboró la encuesta definitiva, basada en preguntas cerradas en las que el entrevistado elegía una respuesta o varias entre unas categorías de respuestas predeterminadas (Alvira-Martín, 2004; García-Jiménez; Gil-Flores, 1996; Díaz-de-Rada, 2005; IgartuaPerosanz, 2006).

El diseño y envío del cuestionario definitivo y la recopilación de datos se hizo a través de la aplicación para gestión de encuestas online Surveymonkey. El cuestionario constó de 11 preguntas cerradas distribuidas en 3 temas diferenciados:

- datos profesionales y personales: preguntas 1-5.

- experiencia: 6-8.

- formación idónea: 9-11.

\subsection{Trabajo de campo}

Los cuestionarios comenzaron a enviarse mediante correo electrónico el 18 de mayo de 2014 y la encuesta estuvo abierta hasta el 18 de octubre de 2014. El trabajo de campo duró cinco meses en los que se realizaron 4 fases de envío (tabla 1 ).

Tabla 1. Etapas de desarrollo de la encuesta

\begin{tabular}{|l|l|l|l|}
\hline \multicolumn{1}{|c|}{ Número de envío } & \multicolumn{1}{|c|}{ Fecha de envío } & \multicolumn{1}{c|}{ Contenido } & Destinatarios \\
\hline Envío 1 & $\begin{array}{l}18 \text { de mayo } \\
2 \text { y } 18 \text { de junio } \\
2 \text { y } 18 \text { de julio }\end{array}$ & $\begin{array}{l}\text { Presentación de la investigación y } \\
\text { enlace para la encuesta }\end{array}$ & $\begin{array}{l}\text { Toda la BBDD de contactos de agencias que realizan } \\
\text { funciones de CM }\end{array}$ \\
\hline Envío 2 & $\begin{array}{l}2,18 \text { y } 31 \text { de julio } \\
18 \text { de agosto }\end{array}$ & Recordatorio y enlace para la encuesta & Contactos que no habían respondido al envío 1 \\
\hline Envío 3 & 9 de septiembre & Recordatorio y enlace para la encuesta & Contactos que no habían respondido a los envíos 1 y 2 \\
\hline Envío 4 & 9 de octubre & Recordatorio y enlace para la encuesta & Contactos que no habían respondido a los envíos 1, 2 y 3 \\
\hline
\end{tabular}




\section{Análisis de resultados}

\subsection{Datos profesionales y personales}

Hay una mayoría de mujeres frente a los hombres $(61,7 \%$ y $38,3 \%$ respectivamente) y el $96 \%$ es de nacionalidad española. El 50\% tiene entre 25 y 35 años (el porcentaje exacto es $25,8 \%$ para la franja situada entre los 25 y los 30 y $24,2 \%$ para los que se hallan entre los 31 y 35). Entre 36 y 40 años tiene el $18,0 \%$ de los encuestados, entre 41 y 45 el 10,9\% y más de 46 el $14,1 \%$. Solamente el 7,0\% declara tener menos de 25 años.

Esta falta de menores de 25 años se corresponde con el nivel de estudios que poseen, universitarios en su gran mayoría. Un escaso $4,7 \%$ no ha pasado por la universidad, un $3,1 \%$ ha alcanzado titulación en grados superiores y un $1,6 \%$ se quedó en los estudios de bachillerato. Estudios de 2ㅇ ciclo universitario son los que tienen la mayoría de los entrevistados, licenciatura en el $50,8 \%$ de los casos y grados en el $6,3 \%$. Las edades anteriormente reflejadas ponen de manifiesto que aún no ha dado suficiente tiempo para que los egresados de los estudios de grados puedan ser una mayoría, y esto es lógico ya que las primeras promociones de grados en España corresponden a los cursos 2008-2012. Pero esto no implica que todos los estudios hayan implementado los grados en esta fecha, la incorporación de este plan de estudios ha sido progresiva desde 2008. Se observa también un pequeño porcentaje del $2,3 \%$ de profesionales que ha estudiado diplomaturas. Asimismo casi el 30,5\% tiene estudios de tercer ciclo (masters, cursos de extensión, postgrados...), y el 5,5\% ha realizado estudios de doctorado.

La gran mayoría de los encuestados ha estudiado ciencias de la comunicación, un 77,3\% de ellos. La carrera que sobresale es periodismo, cursada por el $50 \%$; le siguen publicidad y relaciones públicas $(21,1 \%)$ y comunicación audiovisual $(6,3 \%)$. Es la misma relación existente entre egresados de periodismo y su posterior colocación en agencias de comunicación como ejecutivos de cuentas. Si nos fijamos en otras áreas observamos las siguientes cifras: administración y dirección de empresas un $10,2 \%$, marketing un $7,1 \%$, informática un $3,9 \%$ y diseño el $1,6 \%$.

\subsection{Experiencia previa}

La mayoría (el 77,6\%) presenta una experiencia de entre 1 y 5 años desempeñando funciones como CM. Concretamente el $28,5 \%$ lleva entre 1 y 2 años y el 49,1\% entre 2 y 5 años. Por tanto casi la mitad de la muestra tiene recorrido profesional en este campo. El 9,5\% manifiesta que ejecuta estas tareas desde entre los 5 y 10 años anteriores. Estas cifras tienen relación con la media de edad mayoritaria que veíamos anteriormente. Si el

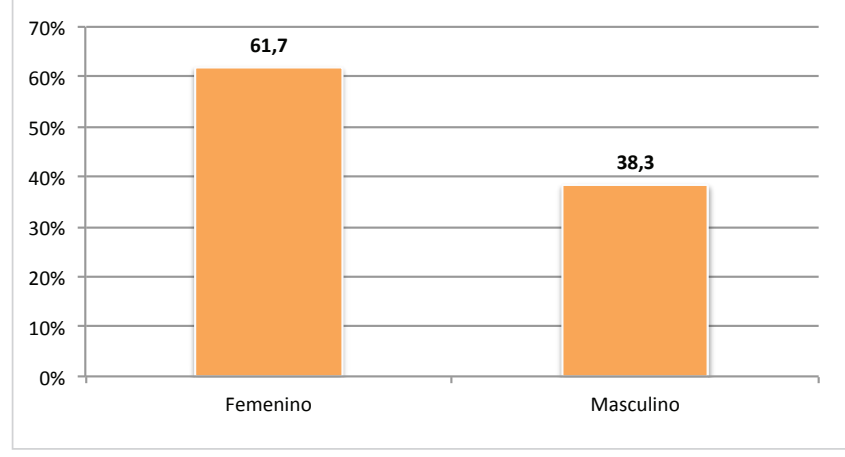

Figura 3. Pregunta 1: sexo

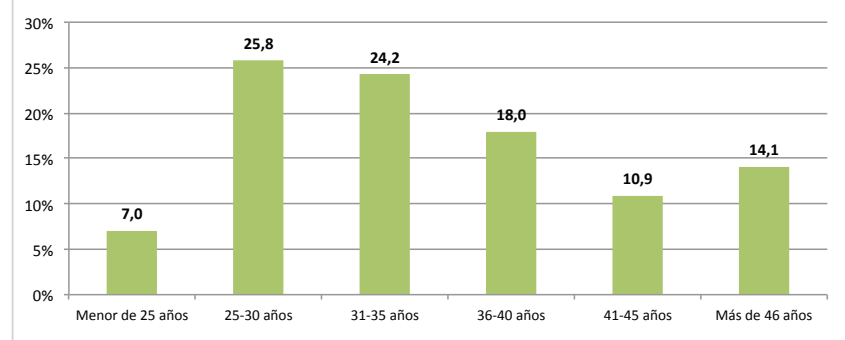

Figura 4. Pregunta 2: edad

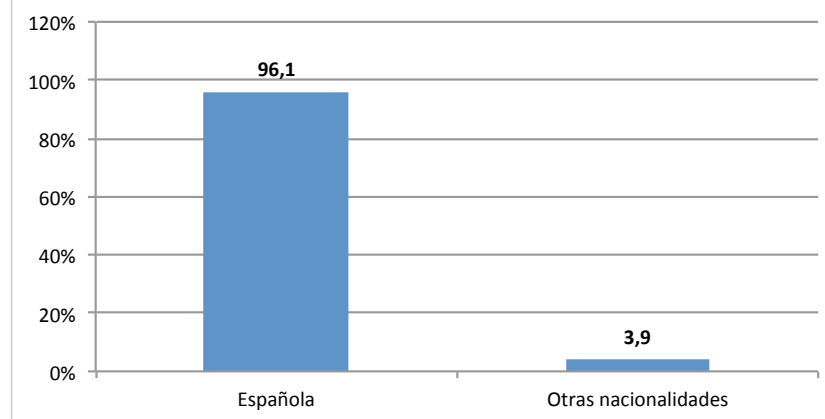

Figura 5. Pregunta 3: nacionalidad

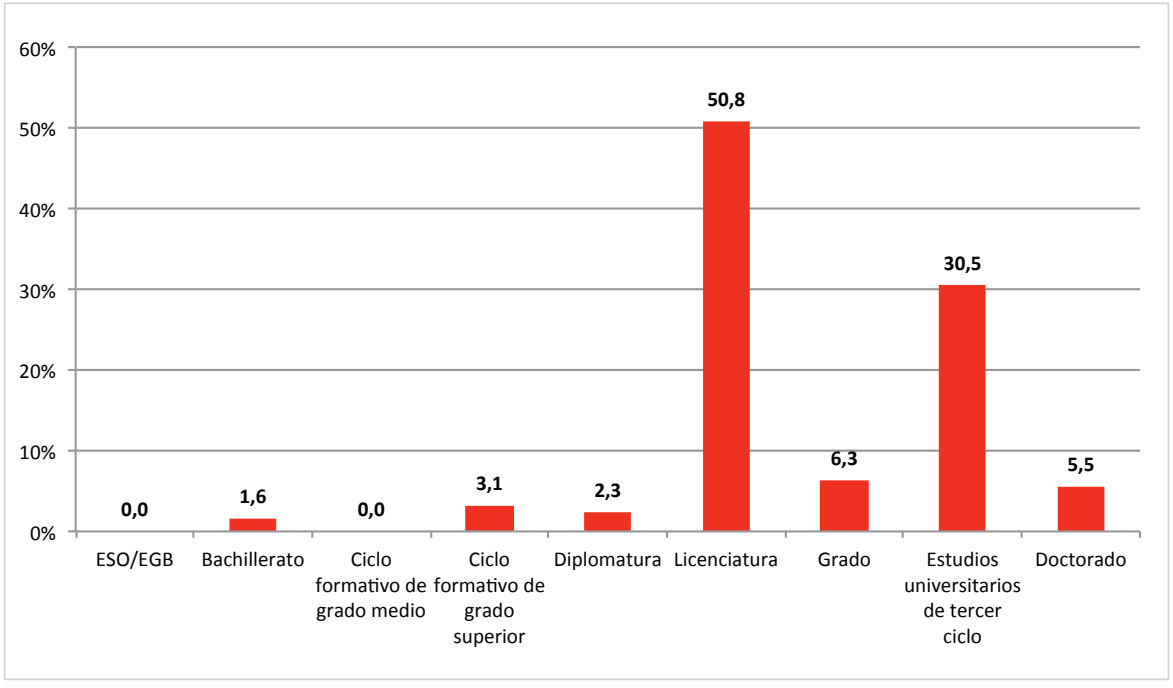

Figura 6. Pregunta 4: nivel de estudios alcanzado 
grueso de ellos tiene entre 25 y 35 años, su experiencia como $C M$ ha de rondar entre los 2 y los 10 . Aunque estas funciones no eran realizadas de forma generalizada por las agencias españolas antes de 2008 , hay un $2,6 \%$ de profesionales con más de 10 años de recorrido en estas gestiones. En el extre-

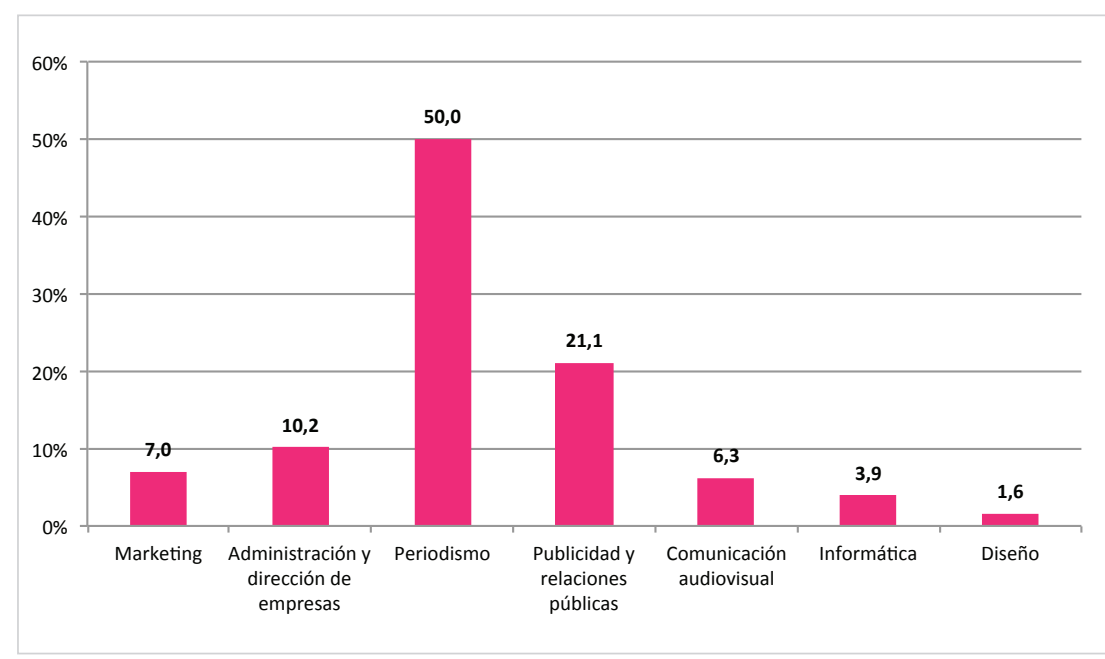

Figura 7. Pregunta 5: área de estudios realizados

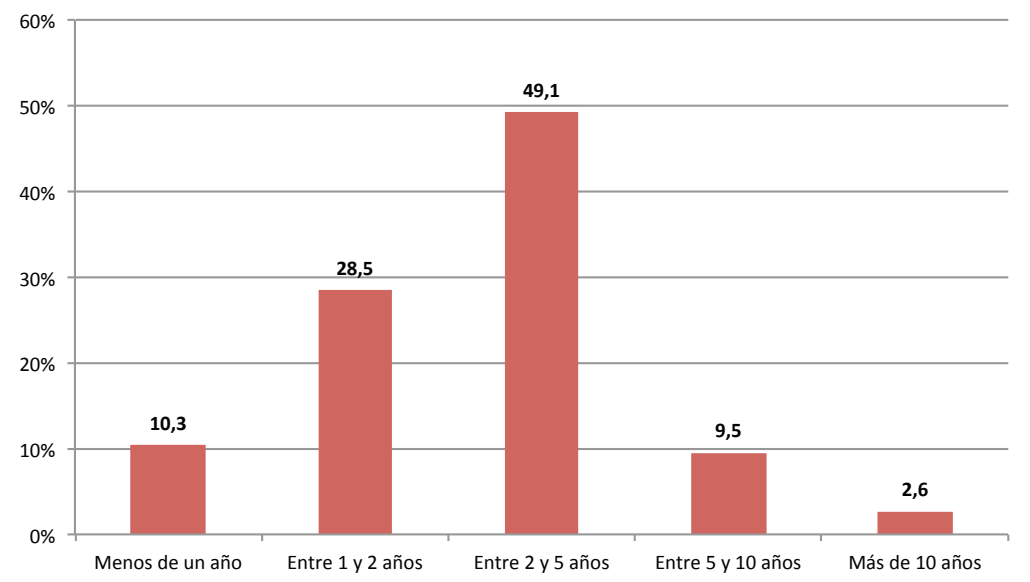

Figura 8. Pregunta 6: años de experiencia como $C M$ mo opuesto se encuentran los casos encuestados con menos de un año de experiencia, el 10,3\%.

En referencia al ámbito profesional en el que han trabajado antes de introducirse en la community management queda patente que la mayoría de ellos proviene del sector de las agencias, como devela el $43,27 \%$ -agencias de publicidad y relaciones públicas $(19,0 \%)$, agencias de comunicación (25\%). Un 26,72\% proviene del entorno de los medios de comunicación.

Por su parte hay un $19,8 \%$ de encuestados que procede de las empresas clientes de la agencia. El $14,7 \%$ ha trabajado en comunicación y relaciones públicas, el 3,5\% en marketing y el $1,7 \%$ en prensa. En ninguno de los casos han estado en el departamento de publicidad, ni en el de informática.

Nos encontramos con un $9,5 \%$ de la muestra que no tenía experiencia previa y ha comenzado su vida profesional desempeñando funciones de community management y que probablemente sean los casos de las personas con menor edad.

En cuanto a los puestos profesionales online que han ocupado anteriormente, la mitad de ellos han sido redactores digitales $(50,9 \%)$-acercando el perfil al mundo del periodismo nuevamente- $y / o$ gestores de contenidos digitales $48,3 \%$. Destaca también el porcentaje de ex consultores de comunicación online, un $38,8 \%$. Estas dos últimas ocupaciones están muy ligadas a las propias funciones del CM. En menor medida han trabajado como dinamizadores sociales $(16,3 \%)$, analistas web $(13,8 \%)$ y/o expertos SEM/SEO (7,8\%). Por su

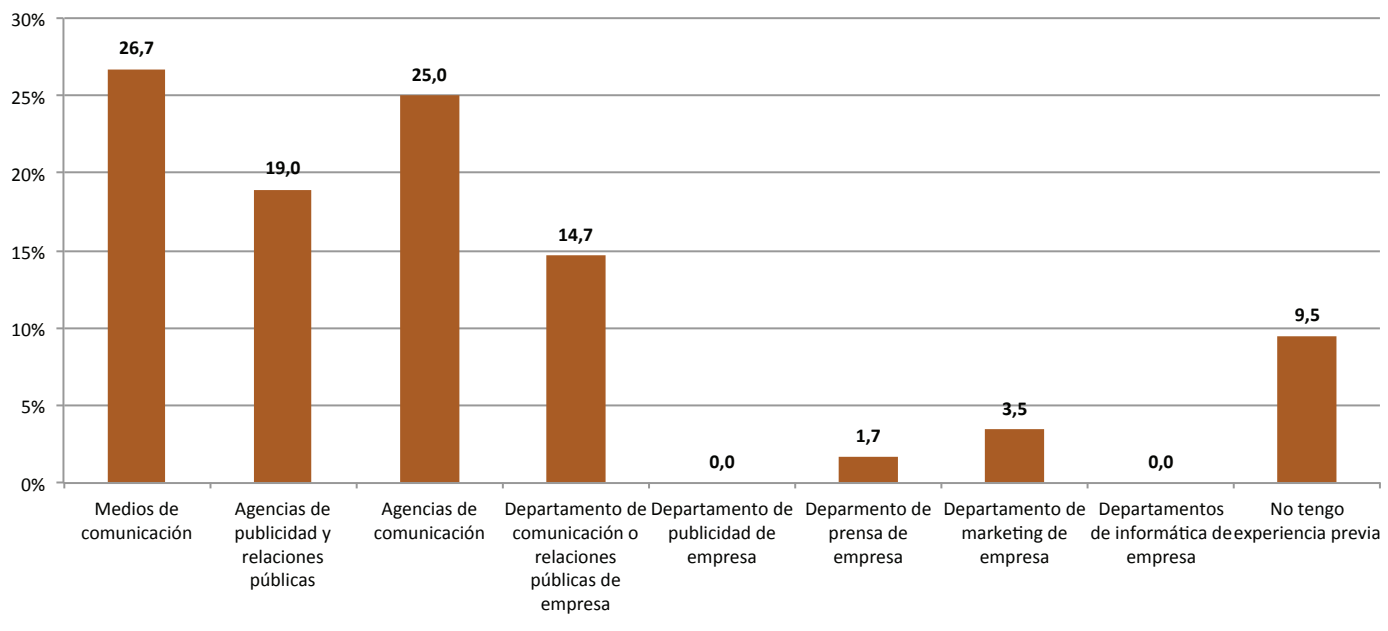

Figura 9. Pregunta 7: ámbito profesional previo en el que desempeñó su carrera antes de incorporarse como $C M$ 
parte un $20,7 \%$ no había desempeñado previamente puestos laborales relacionados con la comunicación online.

\subsection{Formación oportuna para un CM}

Casi de forma unánime están de acuerdo en que se ha de tener formación específica en la materia. Así lo expresó el $89 \%$ de ellos frente al $11 \%$ que no lo estima necesario. El grupo que sí la estiman oportuna opina en un 59,3\% que estos estudios deberían ser de postgrado, un $34,1 \%$ cree que sería suficiente un curso de experto y un $25,3 \%$ de máster. El otro 40,7\% considera que ha de ser durante los estudios de carrera universitaria cuando se adquiera esa formación. El 33,0\% opina que habría que incorporar asignaturas específicas en los planes de estudio de grados concretos y el $7,7 \%$ sugiere que lo más oportuno sería crear grados específicos sobre community management.

Los CM consideran necesaria una formación específica sobre la materia para desarrollar su trabajo, siendo un postgrado la opción más recomendada

En cuanto a los estudios universitarios que creen más idóneos para ejercer como $C M$ vuelven a ser los estudios de comunicación los que más valoración obtienen $(73,5 \%)$. Periodismo es considerada por los $C M$ de agencia la carrera más oportuna, así lo asevera el $42,6 \%$ de ellos, seguida de publicidad y relaciones públicas $(27,7 \%)$. Bastante más atrás se queda comunicación audiovisual, seleccionada por el 3,2\% de la muestra. Los estudios de marketing se perfilan como la tercera opción más válida, seleccionada por el $22,3 \%$. En puestos inferiores quedan administración y dirección de empresas con porcentajes de valoración de 3,2\% y $1,1 \%$ respectivamente.

\section{Discusión de resultados y conclusiones}

La investigación llevada a cabo cumple los objetivos de partida ya que se extraen datos sobre sexo, edad, formación y currículum vitae de los $C M$ de agencias españolas además de describir su trayectoria profesional. Nos encontramos con un perfil compuesto por una mujer de entre 25 y 35 años, que ha estudiado periodismo y cuenta con una experiencia previa de 2 ó 3 años en medios digitales; que además considera imprescindible tener una formación específica en comunicación, especialmente en periodismo y en comunicación digital, para ejercer dicha función.

Estos datos corroboran las hipótesis de partida puesto que se corresponde con el del grueso de las ejecutivas de cuentas de las agencias: mujeres menores de 35 años que han estudiado periodismo. También se verifica que poseen experiencia profesional previa, principalmente en comunicación online.

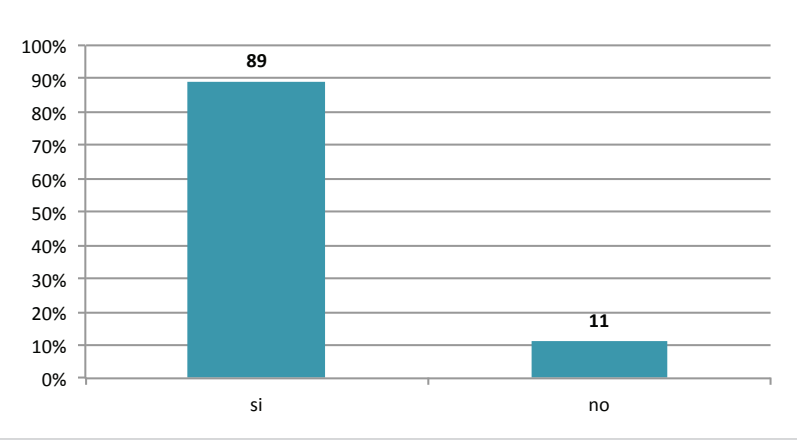

Figura 11. Pregunta 8: puestos relacionados con la comunicación online desempeñados anteriormente

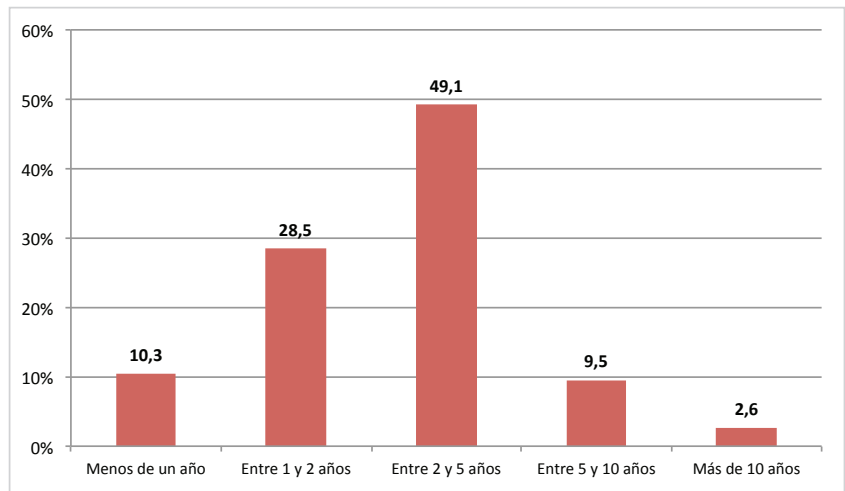

Figura 12. Pregunta 22: tipo de formación específica sobre $C M$ que estima oportuna

Los resultados nos llevan a concluir que la evolución de los CM en el entorno de las agencias repite el patrón de la comunicación corporativa sobre quiénes están desempeñando los trabajos tanto por el perfil como por la experiencia. Se evidencia claramente la estrecha relación entre los CM y el periodismo. Esta relación aleja al $C M$ de aspectos más comerciales y de atención al cliente, propios del marketing; y lo acerca a la idea de relaciones públicas -enfoque de este trabajo- con una perspectiva más informativa y de gestión del contenido. 


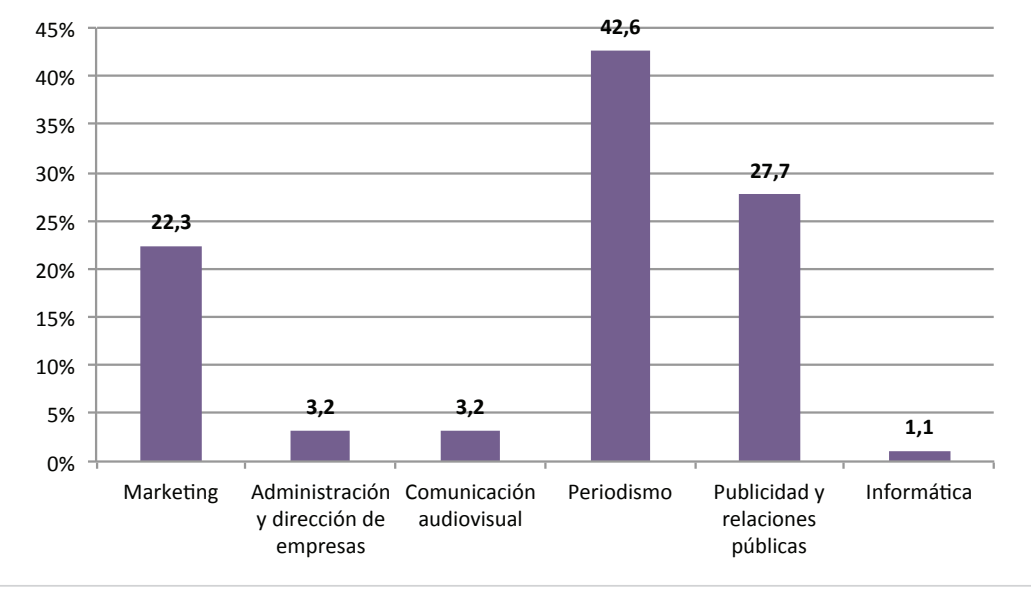

Figura 13. Pregunta 23: estudios más oportunos para ejercer como CM
Almansa-Martínez, Ana; Godoy-Martín, Francisco-Javier (2012). "El community manager en las principales empresas de España: una aproximación a su formación y su situación laboral". Estudios sobre el mensaje periodístico, v. $18, \mathrm{n}$. especial octubre, pp. 57-65.

http://dx.doi.org/10.5209/rev_ESMP.2012. $v 18.40887$

Alvira-Martín, Francisco (2004). La encuesta: una perspectiva general metodológica. Madrid: CIS. ISBN: 9788474765564

Buendía-Eisman, Leonor; Colás-Bravo, María-Pilar; Hernández-Pino, Fuensanta (1998). Métodos de investigación en psicopedagogía. Madrid: McGraw-Hill. ISBN: 978 8448112547

La investigación ha puesto de manifiesto la necesidad de formar específicamente a los CM y cómo ellos mismos lo estiman necesario para llevar a cabo su labor. Es por ello que las facultades con estudios de comunicación deben abordar cuál es la mejor fórmula para resolver esta demanda del mercado laboral.

Para obtener una radiografía más completa de estos profesionales sería interesante ahondar en otros aspectos como tipo de contratos, funciones concretas que llevan a cabo o aspectos referentes al mercado laboral como su movilidad - cada cuánto tiempo cambian de trabajo, fidelidad con la agencia u organización- y la adaptación tecnológica que se ha requerido.

A pesar de la relevancia de esta figura, existe poca bibliografía científica sobre los $C M$ y por el contrario se ha publicado gran cantidad de manuales y libros de uso profesional

Estimamos oportuno que se lleven a cabo estudios semejantes de forma periódica para poder analizar la progresión que se produce en la profesión a corto, medio y largo plazo; así como ampliar el estudio a los CM que trabajan en empresas e instituciones. En el primero de los casos se podrá ver qué ocurre cuando las agencias tengan en sus plantillas una mayoría de nativos digitales y egresados de los grados del Plan Bolonia. Con la segunda propuesta se comprobarían similitudes y diferencias con el mundo de las agencias y así llegar hasta datos globales del estado de la community management en España.

\section{Bibliografía}

Aced, Cristina (2013). Relaciones públicas 2.0. Cómo gestionar la comunicación corporativa en el entorno digital. BarceIona: Editorial UOC. ISBN: 9788490292495

Adecec (2008). La comunicación y las relaciones públicas en España: radiografía de un sector. Madrid: Adecec.

http://prnoticias.com/images/stories/comunicacion/ARCHIVOS/ presentacin_del_estudio_adecec.pdf
Castellblanque, Mariano (2001). Estructura de la actividad publicitaria: la industria de la publicidad de la A a la Z. España: un caso extrapolable. Barcelona: Paidós Ibérica. ISBN: 9788449310553

Castelló-Martínez, Araceli (2010a). La figura del community manager.

http://goo.gl/ud4iHa

Castelló-Martínez, Araceli (2010b). “Una nueva figura profesional: el community manager". Revista pangea, n. 1, pp. 74-97. http://dialnet.unirioja.es/servlet/articulo?codigo $=3405401$

Castelló-Martínez, Araceli (2012). "El estudio de la comunicación publicitaria on-line en el grado en publicidad y RRPP". Questiones publicitarias, v. 1, n. 17, pp. 1-23. http://goo.gl/zkPU5N

Cobos, Tania-Lucía (2011). "Y surge el community manager". Razón y palabra, n. 75.

http://www.razonypalabra.org.mx/N/N75/varia_75/ varia2parte/15_Cobos_V75.pdf

Díaz-de-Rada, Vidal (2005). Manual de trabajo de campo en la encuesta. Madrid: CIS. ISBN: 9788474763881

Diezhandino, María-del-Pilar (2012). El periodista en la encrucijada. Madrid: Ariel; Fundación Telefónica. ISBN: 978 8408008248

http://goo.gl/ZpQksw

Dircom (2010). El estado de la comunicación en España 2010. http://goo.gl/AZrnhU

Elorriaga-Illera, Angeriñe (2013). El marketing y las redes sociales: la figura del community manager en las empresas de la Comunidad Autónoma Vasca y la Comunidad Foral de Navarra. Tesis doctoral, Universidad del País Vasco.

http://www.slideshare.net/angetxu/presentacin-tesisdoctoral-sobre-el-community-manager

FTI, Fundación Tecnologías de la Información (2011). Perfiles profesionales más demandados en el ámbito de los contenidos digitales en España 2012-2017. Madrid: FTI, Fondo Social Europeo.

http://ametic.es/sites/default/files/pafet_vii_perfiles_ profesionales_cd_ti-rooter_1.pdf 
García-Jiménez, Eduardo; Gil-Flores, Javier (1996). Metodología de la investigación cualitativa. Málaga: Ediciones Aljibe. ISBN: 9788487767562

Godoy-Martín, Francisco-Javier (2012). "La figura del community manager en las instituciones públicas andaluzas". En: Actas 70 Congreso internacional de investigación en relaciones públicas. Relaciones públicas: el diálogo de las organizaciones, Sevilla, 21-23 marzo, pp. 56-68.

http://airrpp.org/descargas/Actas_VII_Congreso_AIRP_ Sevilla2012.pdf

IAB (2011). Observatorio del mercado laboral de los profesionales del marketing, la comunicación y la publicidad digital. 2a oleada, junio.

http://goo.gl/q5crX9

Igartua-Perosanz, Juan-José (2006). Métodos cuantitativos de investigación en comunicación. Barcelona: Bosch. ISBN: 9788497902717

Keath, Jason (2013). “The 2013 community manager report infographic". Socialfresh, 28 enero.

https://www.socialfresh.com/community-managerreport-2013

Noguero-Grau, Antonio (1995). La función social de las relaciones públicas: historia, teoría y marco legal. Barcelona: EUB-ESRP. ISBN: 9788489607163

RegionsJob (2014). Étude: les community managers en France. http://goo.gl/1xgVvG

Sanz-Martos, Sandra (2012). “¿Por qué lo llaman content curator cuando quieren decir documentalista?" COMeIN. Revista de los estudios de ciencias de la información y comunicación UOC, n. 10.

http://www.uoc.edu/divulgacio/comein/es/numero10/ articles/Article-Sandra-Sanz.html

Silva-Robles, Carmen (2012). "Community managers: la dirección de RRPP en la Red". Revista internacional de relaciones públicas, v. 2, n. 3. pp. 193-216.

http://dx.doi.org/10.5783/RIRP-3-2012-10-193-216

\section{No descuides mantener actualizados tus datos en el Directorio EXIT (EXpertos en el Tratamiento de la Información)}

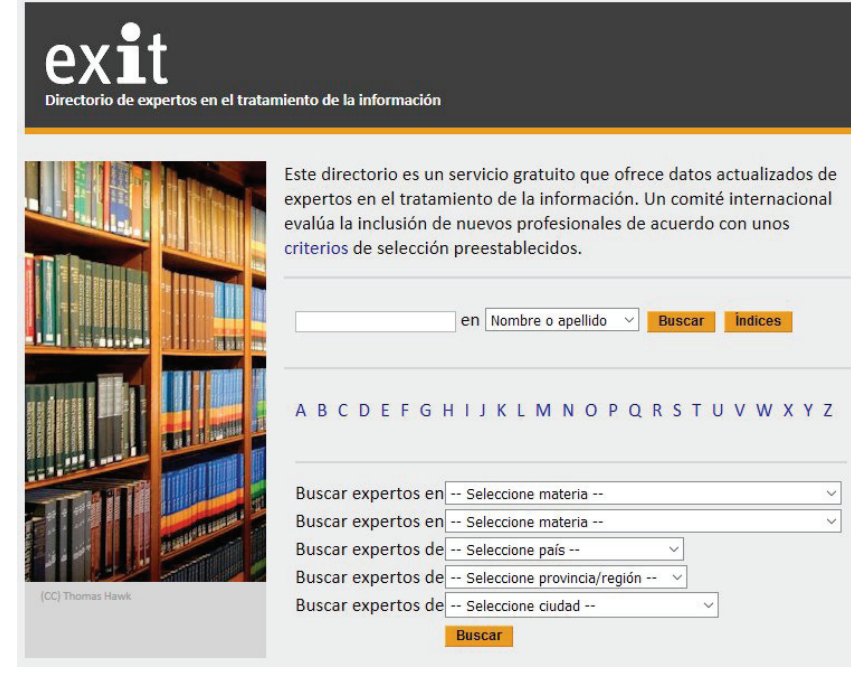

Países
España
Colombia
México
Argentina
Brasil
Reino Unido
Cuba
Perú
Chile
EUA
Italia
Portugal
Francia
Holanda
Ecuador
Venezuela
Bélgica
Alemania
Uruguay
Suiza

$\triangle$ curriculum no
Puedes incluir enlaces a tus páginas de las redes sociales

Especialidades más representadas en EXIT $\quad n^{\circ}$

Gestión de la información y del conocimiento 942

Web 2.0 y redes sociales 747

Biblioteca universitaria $\quad 706$

Recuperación de información y búsquedas 636

Información científico-técnica $\quad 577$

Biblioteca digital 550

Planificación y gestión $\quad 483$

Bibl. especializada / Centro de documentación 454

Análisis y diseño de sistemas de información 453

Gestión de contenidos $\quad 437$

Open access $\quad 427$

Comunicación 422

Catalogación, clasificación e indexación $\quad 401$

Bases de datos (contenidos) 388

Educación, formación, alfin 337

Revistas electrónicas $\quad 335$

Bibliometría y cibermetría $\quad 324$

Marketing y promoción 317

Documentación en general 316

Arquitectura de la información 311

Servicios de internet en general $\quad 297$

Información biomédica $\quad 290$

Diseño de webs 284

Usabilidad, interfaces e interacción $\quad 274$

Biblioteca pública 270

Software documental $\quad 245$

Ontologías, metadatos, taxonomías y tesauros 230

Periodismo $\quad 229$

Multimedia y documentación audiovisual 229

$\begin{array}{ll}\text { Información humanística } & 227\end{array}$

\section{http://directorioexit.info}

